\title{
Simulated Sensitivity Improvement of Optical Receiver in Fiber Optic Network
}

\author{
Elechi Promise, Orike Sunny, Minah-Eeba Winner \\ Department of Electrical Engineering, Rivers State University, Port Harcourt, Nigeria
}

Email address:

elechi.promise@ust.edu.ng (E. Promise)

\section{To cite this article:}

Elechi Promise, Orike Sunny, Minah-Eeba Winner. Simulated Sensitivity Improvement of Optical Receiver in Fiber Optic Network. Advances in Applied Sciences. Vol. 3, No. 4, 2018, pp. 43-51. doi: 10.11648/j.aas.20180304.11

Received: September 12, 2018; Accepted: September 20, 2018; Published: October 15, 2018

\begin{abstract}
With the increasing demand and growth in optical telecommunication networks, the sensitivity of an optical receiver become an important part of telecommunication transmission networks. The unlimited bandwidth of an optical fiber create a path for large volume of signals to flow rapidly in a fraction of a seconds. This increase in the volume of transmitted signals causes severe traffic congestion, making the receiver insensitive, prolong the signal processing time and identified bits wrongly. In this research, Optisystem 7 professional software using Erbium Doped Fiber (EDF), Avalanche photodiode (APD), Non-Return-to-Zero modulation format, optimization tool and low pass Bessel filter, CW laser, pump laser, a gain flattering filter, attenuator, Bit Error Rate Analyzer (BER) etc, was used to improve the sensitivity of the receiver. The procedure of the design was to first improve on the gain using Erbium Doped Fiber Amplifier (EDFA), reduce loss and propagation time and increase the signal flow. Optisystem simulation was used to determine the optimum gain and the receiver sensitivity for several values of attenuation. This research investigated and analyzed the performance of the optical receiver at a wavelength of $1550 \mathrm{~nm}$, optic fiber of length $100 \mathrm{~km}$. Simulations were made and the Bit Error Rate analyzer values taken. Several values of Q-Factor and Bit Error Rate were tabulated and the corresponding graphs plotted. There was an improvement in the gain, quality factor, the quality of signal with a low noise value and the extended transmission or propagation time was reduced. The result of the design showed an improvement in the sensitivity of the receiver.
\end{abstract}

Keywords: EDFA, APD, Attenuator, Filter, Optisystem

\section{Introduction}

\subsection{Research Background}

Optical fiber is a major component of several communication organization. It is a fundamental structure of many wide and local area network. In addition, recent advances in the development and manufacture of optical systems has made it the lasted frontier in the field of telecommunication. Optical systems have replaced a lot of copper wire and are also replacing most of the point-to-point long distance communications traffic previously handled by microwave particularly transoceanic. Optical systems are recognized as the superior medium for developing high band width communication signals over long distances [1].

Optical system comprises of three major parts: a fiber optic transmission strand which carries the light signal from a few feet to thousands of kilometers, a source of invisible infrared radiation (optical transmitters), a photo sensitive detector to convert the optical signal back into an electrical signal (optical receiver). Fiber optic data link transmit the input data via fiber optic devices and ensures that the data is available at the output as information. Fiber optic data link perform three functions [2].

The sensitivity of an optical receiver is defined as the minimum average received power (Prec) needed by the receiver to operate at a bit error rate (BER) of 10-9, (the minimum optical power of the incident light signal that is needed to keep the required BER or the minimum energy needed for the receiver to detect an incoming signal). The function of the transmitter is to convert the input signal from the input source to an optical signal for transmission. It comprise of two distinct parts: a source drive circuit and the interface circuit. The drive circuit is responsible for converting the electrical signal into optical signal. It achieved this process by regulating the current that flows through the 
light source. Two major types of optical signal sources are laser diodes and LEDS. The optical signal is send into the fiber by the optical source. The optical signal becomes weaker and gradually distorted as a result of noise, scattering, dispersion and absorption processes in the fiber [3].

\subsection{Review of Related Works}

A major improvement in the field of optical receiver development occurred in 1985 when Lanny S. Smoot came up with the idea of using trans-impedance amplifier (TIA) for optical receivers. TIA is a receiver comprising of a reverse biased ADP or PIN coupled to the input of a high impedance amplifier. His invention relates particularly to optical communication receivers that are used in light guide system, more specifically to the trans-impedance amplifier (TIA). He stated that optical receivers are an aspect of light guide system used for optical communication networks and generally have a few dynamic range [4]

[14] developed a control loop used in the optical receiver within which the dynamic range of the input amplifier was widened. The idea of his invention was to utilize a PIN diode as a regulatory resistance component joined to the input of the trans-impedance amplifier. This was done in order to distinguish the direct voltage component from the voltage on the output of the trans-impedance amplifier, and to regulate the PIN diode resistance on the point of the said direct voltage elements. The advantageous point of his invention is the use of inexpensive components [11]. The problem with his invention is that the addition of a control loop impaired the sensitivity of the optical receiver a little $[5,14]$.

[6] invented optical receiver for WDM free space communication system. The optical receiver consist of a telescopic system having a primary and secondary mirror, a block of beam splitter, a spherical aberration from chromatic components and diaphragm placed along an optical axis of the optical receiver. The chromatic component is used to demultiplex the wavelength channels of the input optical beam of the receiver in such a way that channels are focused at different focal planes on the optical axis of the receivers $[6,13]$.

In 2012, a group of engineers designed an optical receiver using trans-impedance amplifier with light detector and equalization in MOS technology. They combined spatially modulated light (SML) detector with an equalizer in a monolithically integrated photo detector and obtained a maximum data rate. It was the fasted photo detector integrated in a standard CMOS design that uses standard supplies at $3.3 \mathrm{v}$ or below. To increase the functionality of the photo detector, it was implemented in CMOS technology as photodetector with SML detector. This detector consist of a row of photodetector alternatively covered and uncovered with a light blocking metals. The design eliminated extra overhead and cost of assembly of multichip. The problem of Emergency Detection System (EDS), parasitic associated with the bond pad, ground bounce and bond wire were also eliminated $[7,11,12]$.

\section{Materials and Method}

\subsection{Simulation Tools}

Figures 1, 2 and 3 show the main tools and sequence used in optisystem software to simulate the design.

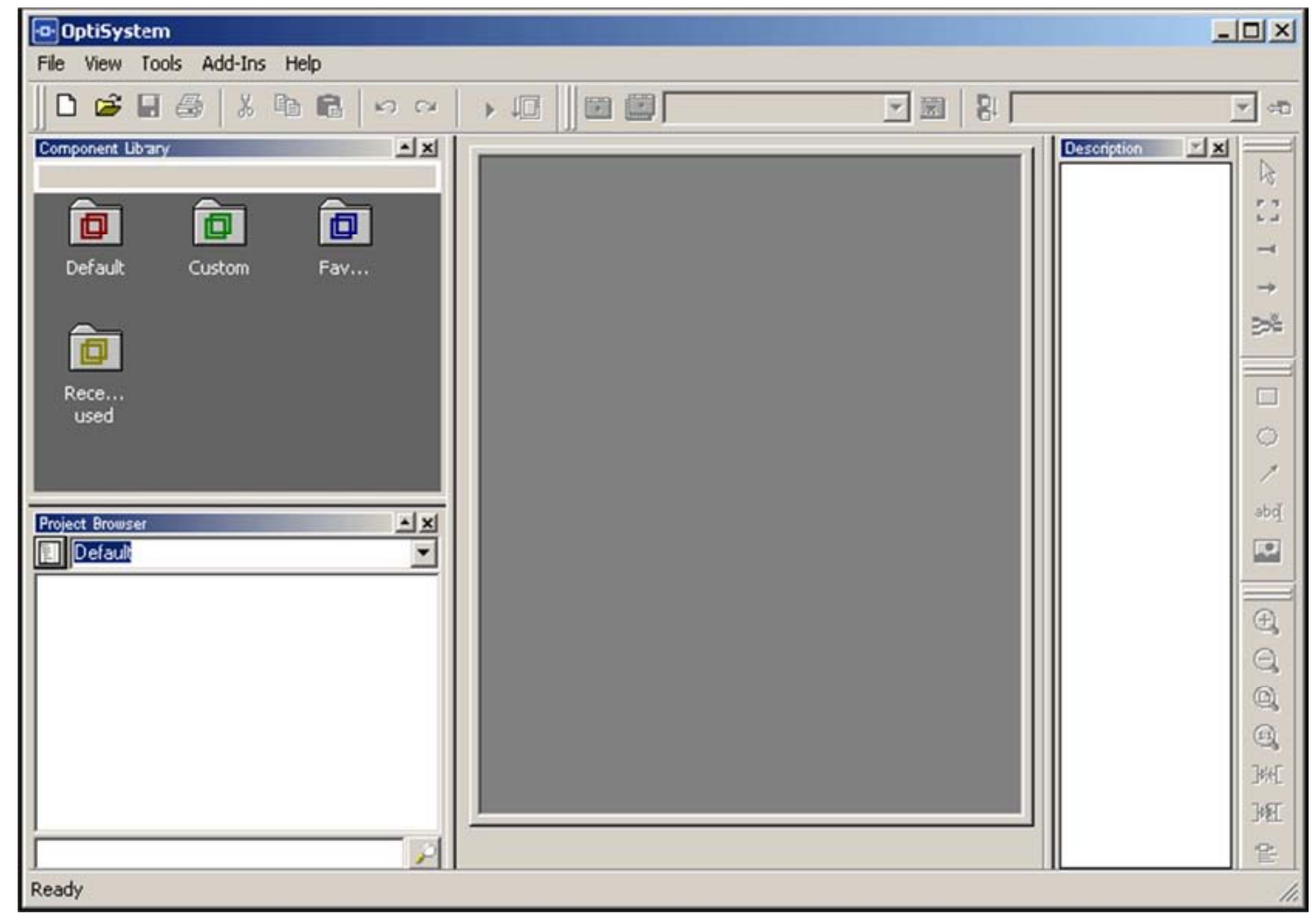

Figure 1. Optisystem Graphical User Interface (GUI). 


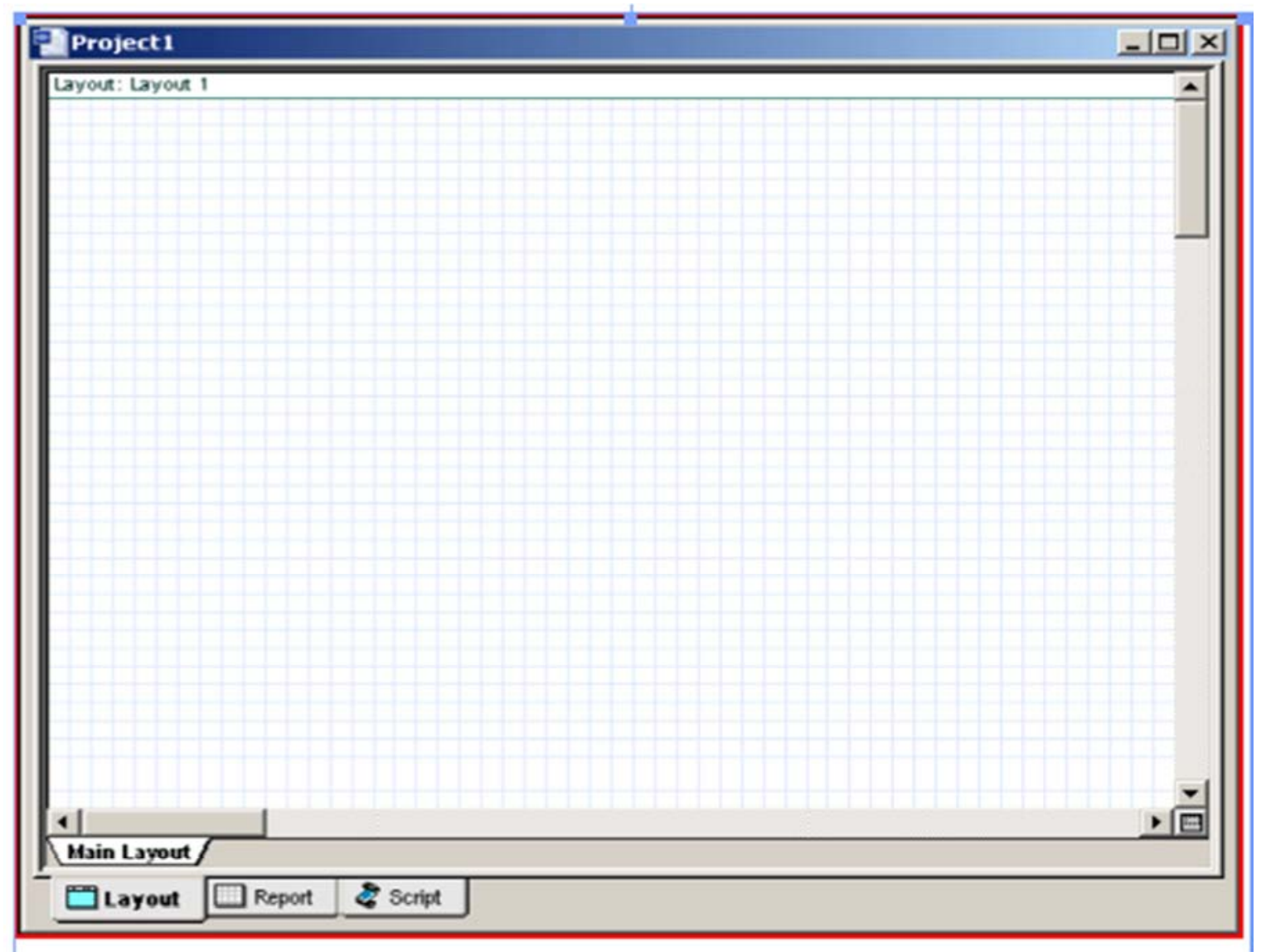

Figure 2. Research Layout Window.

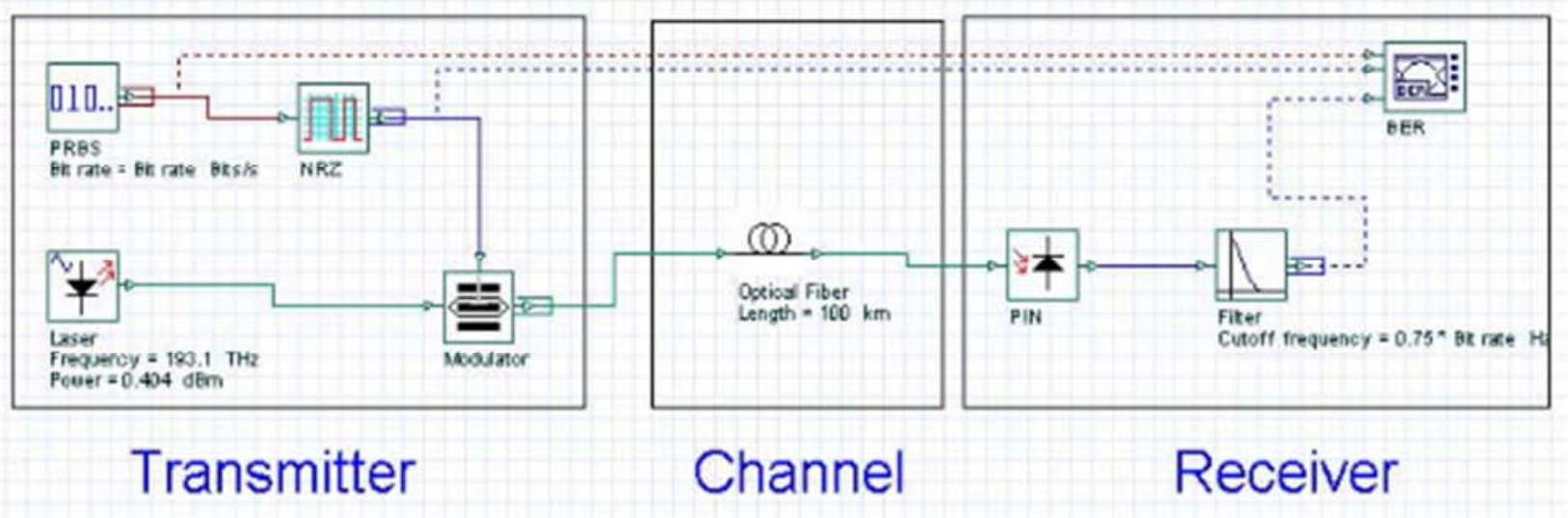

Figure 3. Optisystem Components.

\subsection{Eye Diagram Pattern}

This is a simple and improved measurement techniques to access the performance of an optical telecommunication system. It produces a visual way of monitoring the optical receiver performance; closing of the eye is an indication that the optical receiver is not functioning adequately. When an oscilloscope is coupled and driven by an optical receiver output and is activated by the signal source driving the transmitter, an eye pattern is displayed. The more the eye opens, the better the optical signal quality. The eye diagram is produced in time domain and it permit the effect of waveform distortion to be displayed immediately the simulation is activated as shown in figure. 4. To get the correct system performance, several word patterns should be made available by creating a random data signal to simulate the real situation. The Bit error rate Analyzer measures the performance of the system based on the signal before and after the propagation $[8,11]$. 


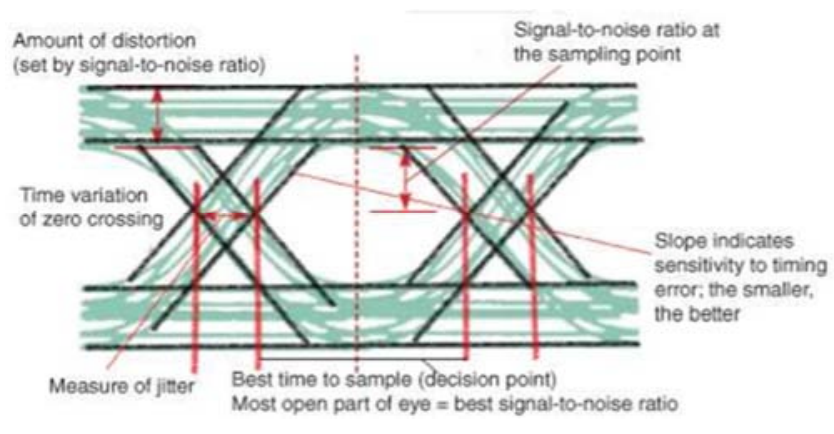

Figure 4. Eye Diagram Interpretation [9].

\subsection{Bit Error Rate}

This is the ratio of detected bit error to the number of total bit transmitted. It is also the probability of total errors in a number of bits of data to the total transmitted bits at a given time interval. The error rate for telecommunication varies from $10^{-9}$ to $12^{-12}$ and does depend on the types of service. The eye diagram analyzer is used to measure the BER in the optical system layout during simulation [10].

\subsection{Description of Method}

The design method used in this research was based on two stages. The design of the Erbium Doped Fiber Amplifier (EDFA), and the optical receiver circuit. The design was carried out using Optisystem 7 professional software.

\subsection{Amplifier Design Circuit}

The Erbium Doped Fiber Amplifier (EDFA) consists of a length of Erbium Doped Fiber, a pump laser (980m, $140 \mathrm{~mW}$ ), a CW laser(155onm, $-30 \mathrm{dBm}$ ) and a component called WDM (lower frequency limit 185Thz, upper frequency limit 200Thz). A pump coupler (bidirectional) combined the optical signal and the pump wavelength so that they can be transmitted at the same time via the Erbium Doped Fiber (EDF, 5m). The EDFA was designed such that the pump energy and the signal propagates in the same direction (forward pumping). ). The optical signal enters the amplifier via the input port or $\mathrm{CW}$ laser, and then passes via a tap whose function is to divert a small amount of the signal power, about 1-2 percent to an input detector (Avalanche photodiode). Greater percentage of the signal from the tap passes via an isolator and then combined with the pump energy emitted by the $980 \mathrm{~nm}$ pump laser diode. The combined optical signal and the pump energy were transmitted along the EDF, where signal amplification took place as shown in Figure 5.

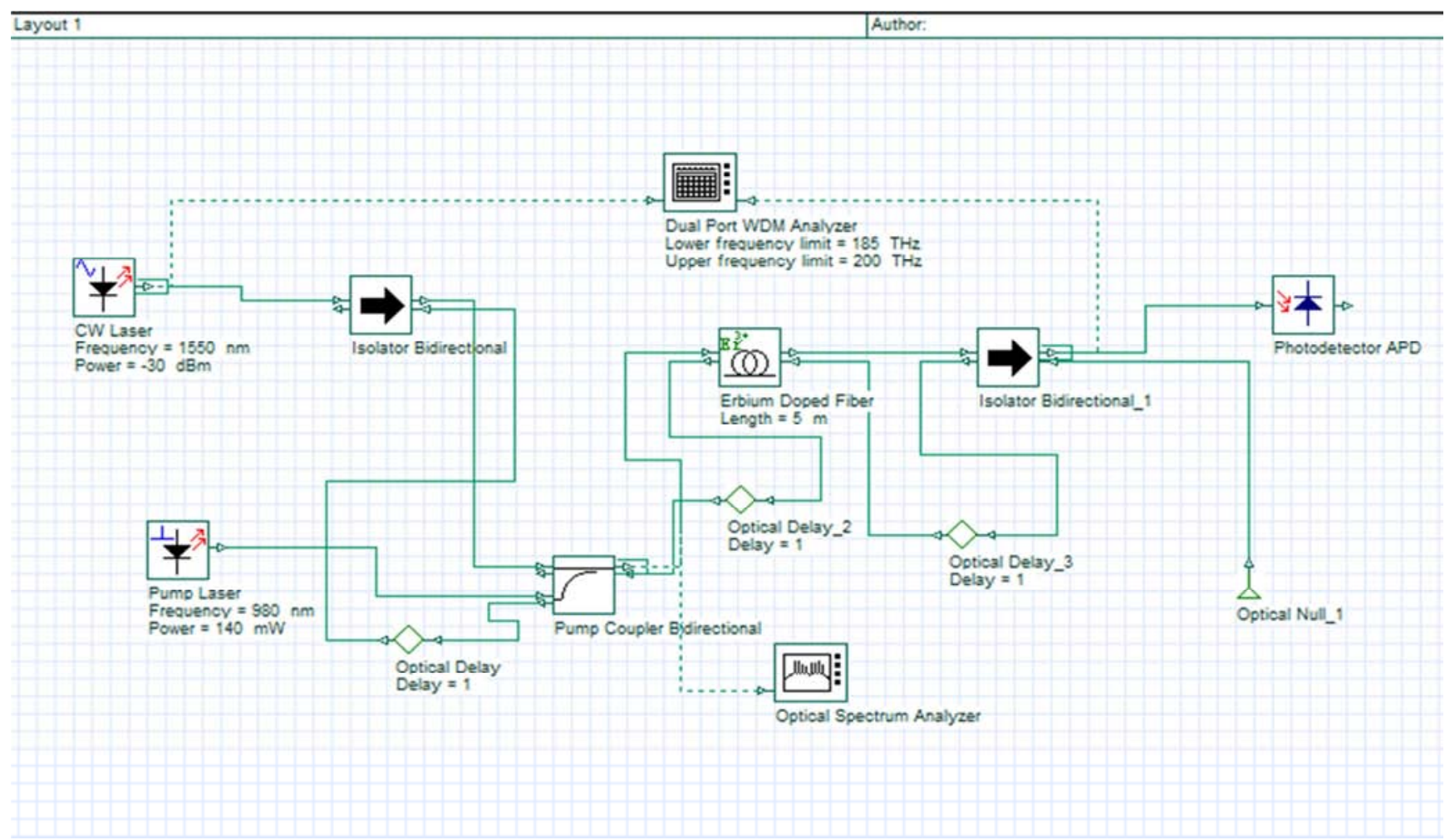

Figure 5. Erbium Doped Fiber Amplifier Design.

\subsection{The Optical Receiver Designed Circuit}

Optical system transmit data using optical carrier wave from a transmitter to a receiver through optical fiber cable. The input signals from the pseudorandom sequence generator contain electrical information that is characterized by 0 's and 1's produced by a NRZ pulse generator. The laser supplies input signal at a wavelength of $1550 \mathrm{~nm}$, the input power was $0 \mathrm{dBm}$ and was modulated at $2.5 \mathrm{GHZ}$ using NRZ pseudorandom binary sequence in a Mach-Zehnder modulator with $40 \mathrm{~dB}$ extinction ratio. The optical fiber cable used in this research was a single mode optical fiber because it yield higher data rate, operate in a long haul distance, and has less dispersion which makes it 
suitable for transmission link. The photodetector diodes translate the optical signal into electrical signal output. The lowpass Bessel filter filters the electrical signals. The system design is shown in Figure 6. Avalanche photodiode was used as the detector because of it high amplification factor. The simulation parameters for the analysis of the receiver sensitivity input power at $100 \mathrm{~km}$ of optical fiber length with various attenuation values were shown.

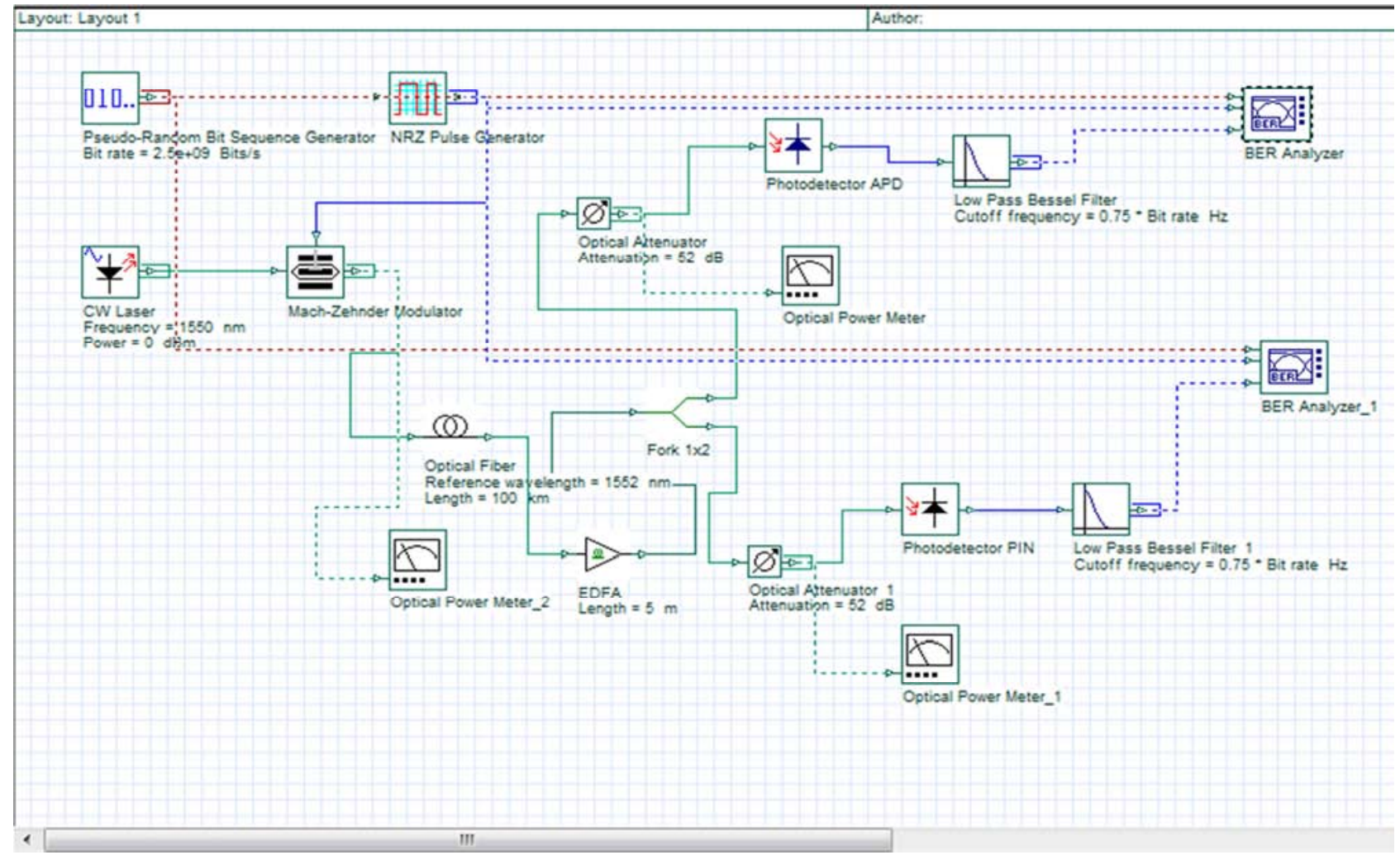

Figure 6. System Design for the Receiver Sensitivity.

\subsection{Visualizer Parameters}

Here, the simulation time window, the number of samples, and the sample rate were defined using three parameters as illustrated in figure. 7 . These parameters include:

- Bit rate

- Bit sequence length

Number of samples per bit

These parameters were used to calculate the Time window, Sample rate, and Number of samples:

Time window $=$ Sequence length $* 1 /$ Bit rate $=1024 * 1 /$ $2.5 \mathrm{E}+9=4.096 \mathrm{E}-7 \mathrm{~s}$

Number of samples $=$ Sequence length $*$ Samples per bit $=$ $1024 * 64=65536$ samples

Sample rate $=$ Number of samples $/$ Time window $=65536$ / $4.096 * 10-7=1.6 \mathrm{E}+11 \mathrm{~Hz}$

\begin{tabular}{|c|c|c|c|c|}
\hline Simulation & Spatial effects & Noise & nal tracing & l \\
\hline Name & \multicolumn{2}{|c|}{ Value } & Units & Mode \\
\hline Simulation window & \multicolumn{2}{|c|}{ Set bit rate } & & Normal \\
\hline Reference bit rate & \multicolumn{2}{|c|}{$\sqrt{\nabla}$} & & Normal \\
\hline Bit rate & \multicolumn{2}{|r|}{2500000000} & Bits/s & Normal \\
\hline Time window & \multicolumn{2}{|r|}{$4096 \mathrm{e}-007$} & $s$ & Normal \\
\hline Sample rate & \multicolumn{2}{|r|}{160000000000} & $\mathrm{~Hz}$ & Normal \\
\hline Sequence length & \multicolumn{2}{|r|}{1024} & Bits & Normal \\
\hline Samples per bit & \multicolumn{2}{|r|}{64} & & Normal \\
\hline Number of samples & \multicolumn{2}{|r|}{65536} & & Normal \\
\hline
\end{tabular}

Figure 7. Simulated values of the Visualizer Parameters.

\section{Results}

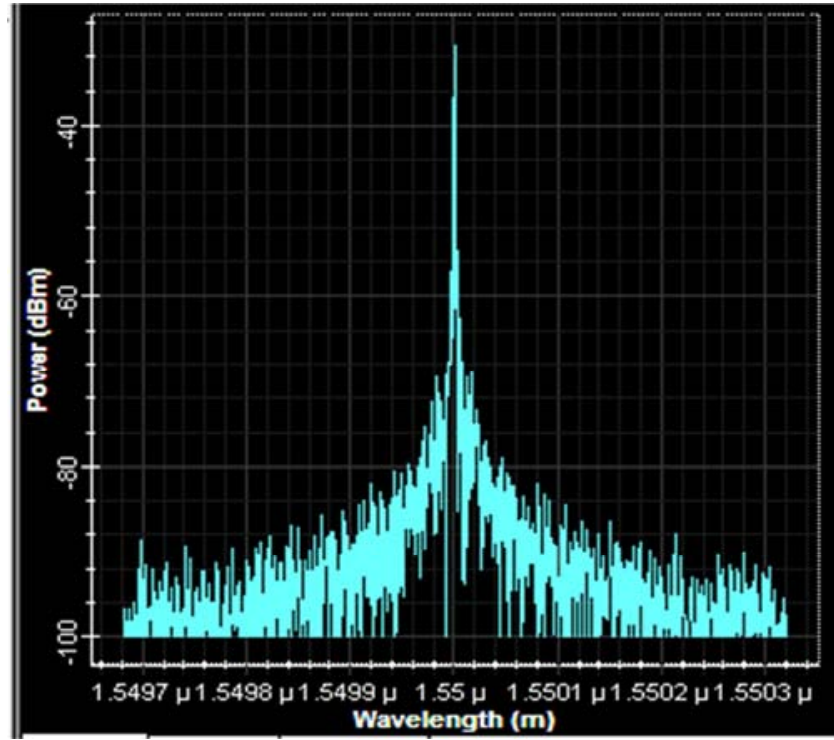

Figure 8. Optical Spectrum Analyzer Showing Power againt Wavelength. 
Table 1. Variation of Power versus Q-factor and BER.

\begin{tabular}{lllll}
\hline $\mathbf{S} / \mathbf{N}$ & Attenuation $\mathbf{( d B / k m )}$ & Receiver power $\mathbf{( d B )}$ & Q-Factor & BER(dB) \\
\hline 1 & 30 & -15.96 & 48.60 & 0 \\
2 & 32 & -17.96 & 48.57 & 0 \\
3 & 34 & -19.96 & 48.48 & 0 \\
4 & 36 & -21.96 & 48.28 & 0 \\
5 & 38 & -23.96 & 47.73 & 0 \\
6 & 40 & -25.96 & 46.43 & 0 \\
7 & 42 & -27.96 & 43.59 & 0 \\
8 & 44 & -29.96 & 38.32 & $1.38 \mathrm{E}-321$ \\
9 & 46 & -31.96 & 30.64 & $2.06 \mathrm{E}-206$ \\
10 & 48 & -33.96 & 22.21 & $1.26 \mathrm{E}-106$ \\
11 & 50 & -35.96 & 15.03 & $2.49 \mathrm{E}-51$ \\
12 & 52 & -37.96 & 9.82 & $4.48 \mathrm{E}-23$ \\
13 & 54 & 39.96 & 6.33 & $1.19 \mathrm{E}-10$ \\
14 & 56 & -41.96 & 4.04 & $2.64 \mathrm{E}-5$ \\
15 & 58 & -43.96 & 0 & 1 \\
\hline
\end{tabular}

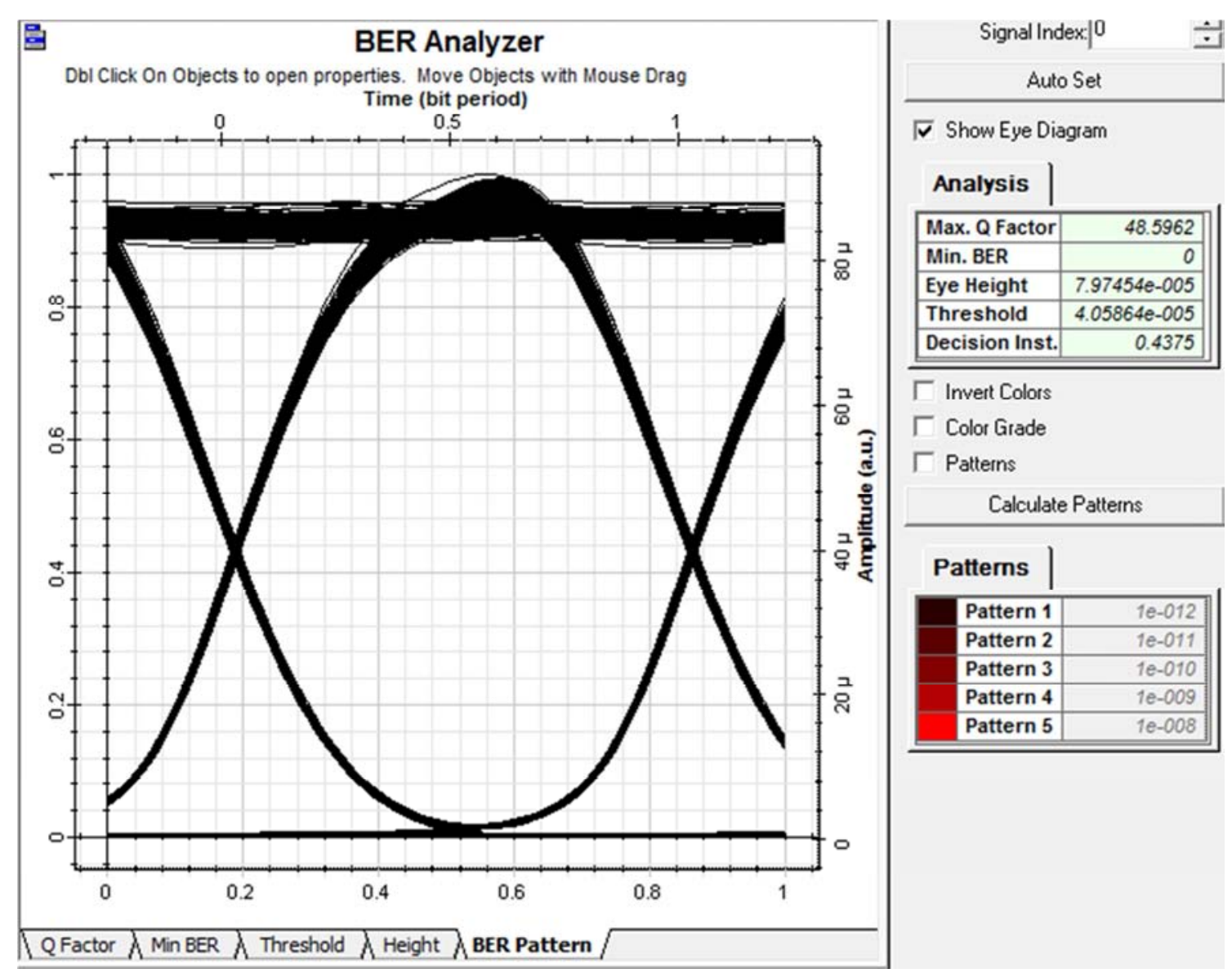

Figure 9. Noiseless Receiver Sensitivity at 30dBm/km.

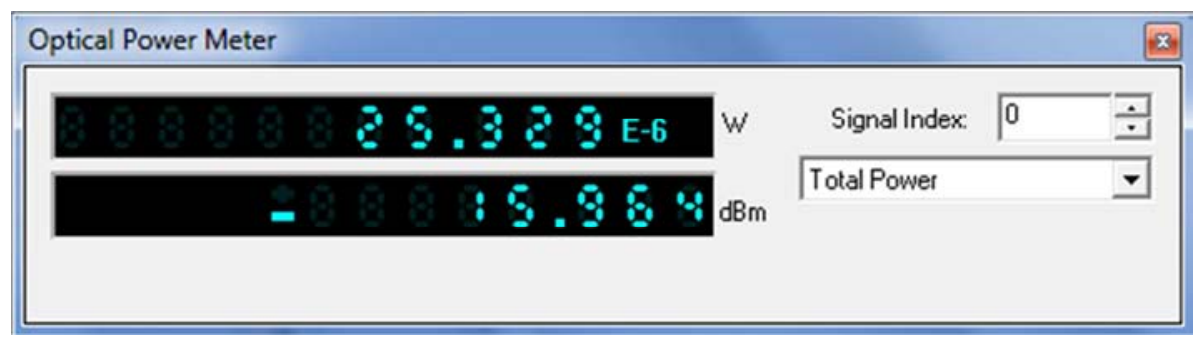

Figure 10. Optical Power at $30 \mathrm{dBm} / \mathrm{km}$. 


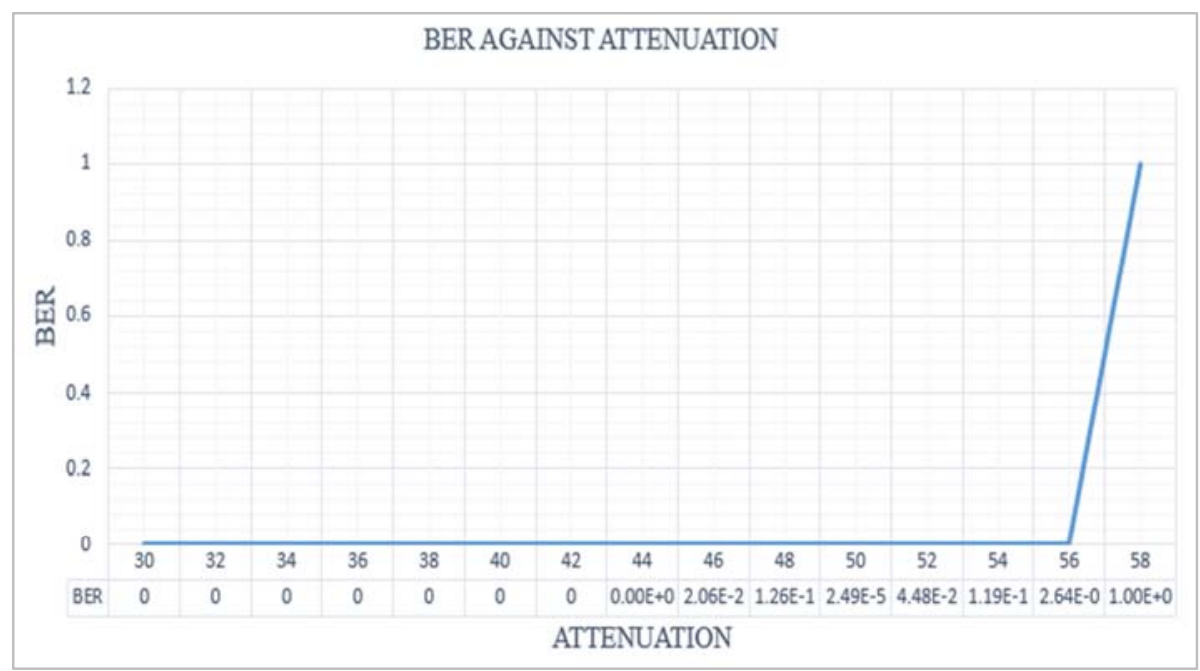

Figure 11. Graph of Attenuation against BER.

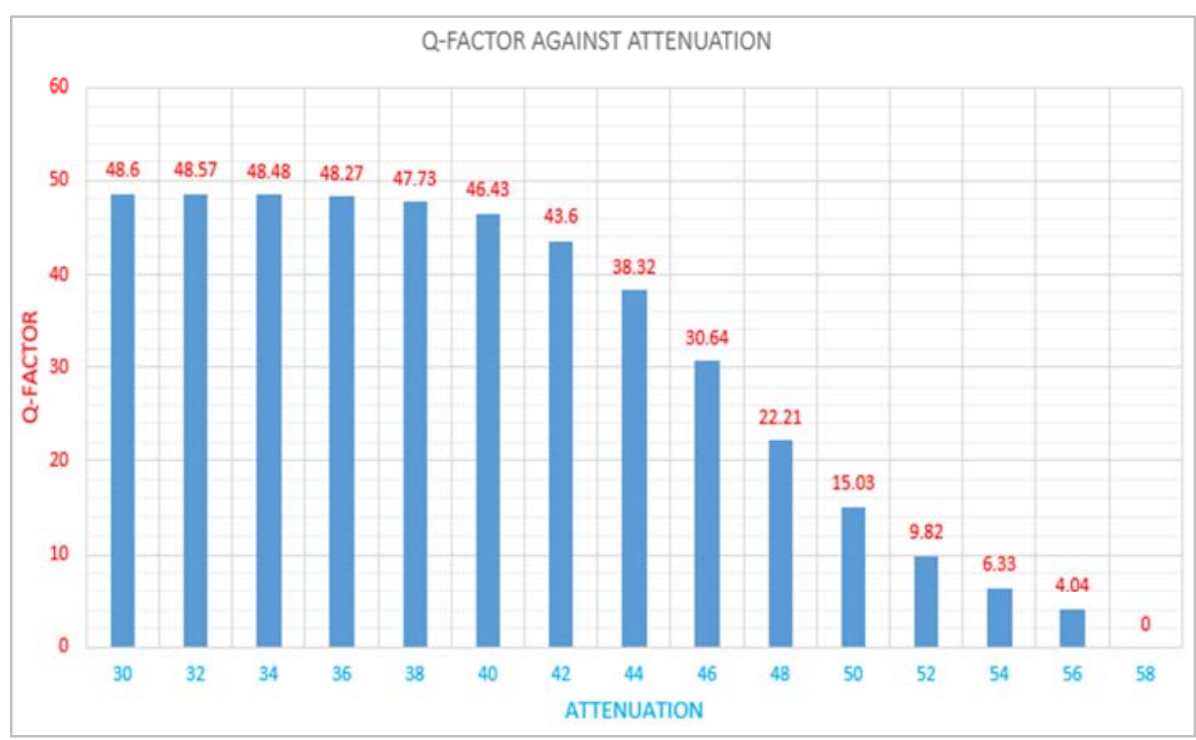

Figure 12. Graph of Attenuation and Q-Factor.

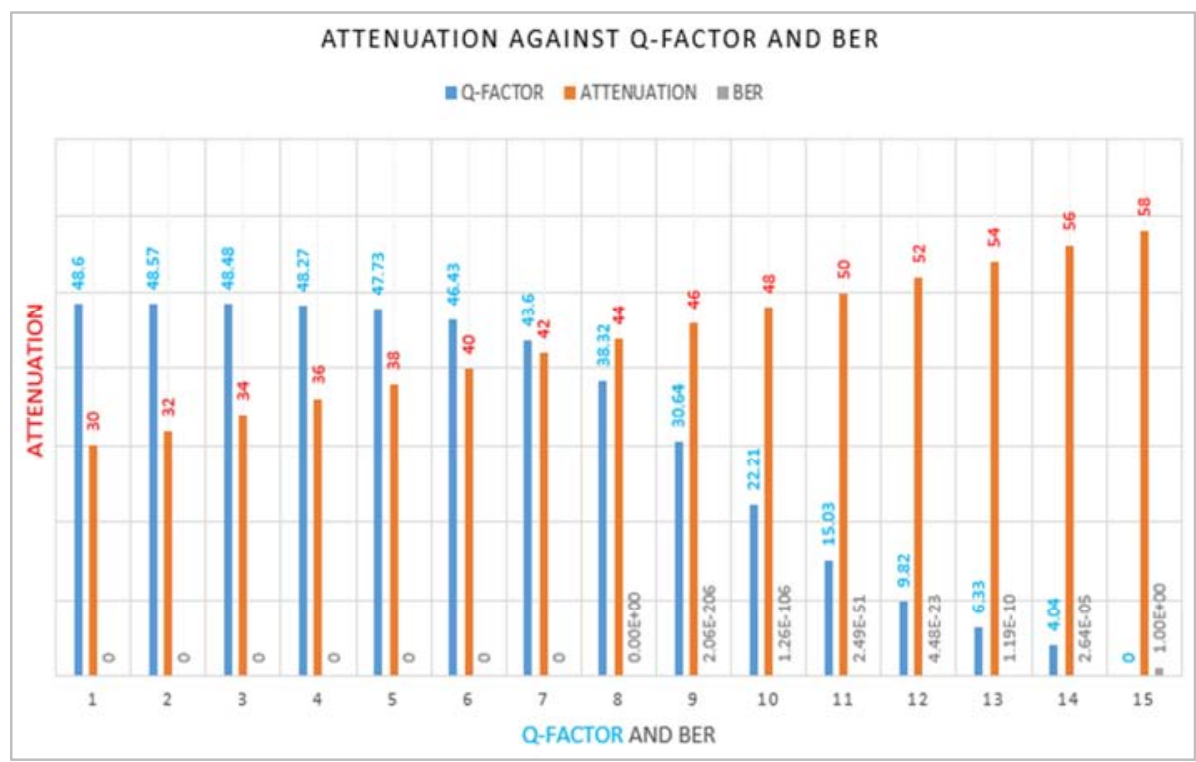

Figure 13. Attenuation versus $B E R$ and $Q$-Factor. 


\section{Discussions}

The Erbium Doped Fiber Amplifier is a very useful amplifier for high frequency communication. It provided better gain and noise value than other optical amplifiers. From the analysis, a better gain of $36.778903 \mathrm{~dB}$ and a noise value of $4.14748 \mathrm{~dB}$ were obtained at $1.55 \mathrm{THz}$ frequency which show that the EDFA has a better gain and noise value at High frequency regions.

Figure 8 shows the results obtained from the optical spectrum analyzer in the optisystem software. It displayed a clear view about the gain flatness for a pump power of $140 \mathrm{~mW}$. The system amplification gain was $36.8 \mathrm{~dB}$ at $1550 \mathrm{~nm}$ band of wavelength with a noise of $4.14 \mathrm{dBm}$.

The following readings were obtained.

Gain $=36.8 \mathrm{~dB}$

Noise Figure $=4.15 \mathrm{dBm}$

Input Signal $=-30.002175 \mathrm{dBm}$

Input OSNR $=23.80414 \mathrm{~dB}$

Output Signal $=6.7767271 \mathrm{~dB}$

Output OSNR $=69.997825 \mathrm{~dB}$

Wavelength (m):

Start Wavelength $=1.54965 \mu$

Centre wavelength $=1.55 \mu$

Stop wavelength $=1.55035 \mu$

Power $(\mathrm{dBm})$ :

Maximum range $=-27.1522$

Minimum range $=-103.469$

Resolution bandwidth

$0.01000 \mathrm{~nm}$

Table 1 shows the variations the received power, the quality factor, and the bit error rate and figures 9 and 10 shows the receiver sensitivity and optical power at 30 $\mathrm{dBm} / \mathrm{km}$ respectively.

The EDFA and the optical receiver system design simulation were done using optisystem simulation software. Several values of Q-Factor and BER against attenuation were obtained and tabulated. The power requirements at different levels of attenuation were also obtained and tabulated. From the simulation and analysis carried out, it was observed that APD detector was sensitive. Figure 11 show that attenuation and BER are directly proportional and the receiver has a BER of 1 at $58 \mathrm{~dB} / \mathrm{km}$. It was observed in Figure 12 that when the attenuation was $54 \mathrm{~dB} / \mathrm{km}$ the Q-Factor was 6.33 which was within tolerance level. Figure 13 show that attenuation and Q-Factor were inversely proportional. From the optimization of the receiver circuit shown in Figure 5, it was observed that the system can cope with attenuation of $54 \mathrm{~dB} / \mathrm{km}$ at a Qfactor of 6.33. From Table 1, it was observed that at an attenuation of $54 \mathrm{~dB} / \mathrm{km}$, the receiver power was $39.96 \mathrm{~dB}$, Qfactor was 6.33, and the BER was 1.19E-10. These values make optisystem very useful for improving sensitivity. The performance of the communication link was optimized at a maximum attenuation of $54 \mathrm{~dB} / \mathrm{km}$ with a Q-Factor of 6.33 as the minimum value needed to improve the sensitivity of the optical receiver optimally.

\section{Conclusion and Recommendations}

\subsection{Conclusion}

The Erbium Doped Fiber Amplifier was used to design the amplifier circuit. It provided better gain and a low noise value than other optical amplifier at high frequency region. It displayed a clear view about the gain flatness for a pump power of $140 \mathrm{~mW}$. The system amplification gain was $36.8 \mathrm{~dB}$ at $1550 \mathrm{~nm}$ band of wavelength with a low noise of $4.14 \mathrm{dBm}$.

By ensuring that the value of the Q-Factor wasn't below 6, optical fiber length maintained at $100 \mathrm{~km}$ with a wavelength of $1550 \mathrm{~nm}$, pump laser at $140 \mathrm{~mW}$ and a gain flat filter that shaped out the noise the OSNR was increased from $23.80414 \mathrm{~dB}$ to $69.997825 \mathrm{~dB}$ while the signal flow was increased from $-30.002175 \mathrm{dBm}$ to $6.7767271 \mathrm{dBm}$.

\subsection{Recommendations}

In view of the simulations and conclusion of the research, the following recommendations are to be considered for continues improved sensitivity.

(1) Maintenance should always be carried out on the communication link to maintain the durability of the fiber.

(2) A qualified professional should regularly carry out functionality inspection on the working condition of the equipment.

(3) The system should be operate under moderate temperature. High temperature could vary the operation of the detector.

\section{References}

[1] Faruk, N., \& Gana, U. M. (2014). Effect of Dispersion on the Performance of an Optical Link. Retrieved from www.intechopen.com. $4^{\text {th }}$ Feb; 2018

[2] Goff, D. (2016). Background of Fiber Optics. Retrieved from www.Fcctests.com/neets.NEETS_MODULE_24_CH_0.1. $30^{\text {th }}$ Jan; 2018.

[3] Goff, D. (2016). Background of Fiber Optics. Retrieved from www.Fcctests.com/neets.NEETS_MODULE_24_CH_0.1.pdf. $30^{\text {th }}$ Jan; 2018.

[4] Ryan, D.K., (2007). Trans-Impedance Amplifier Design using CMOS Technology. Retrieved from www.collectionscanada.gc.ca/obj/s4/f2/dsk3/OKQ/TC-OKQ452.pdf. $7^{\text {th }}$ Feb; 2018.

[5] Tran, (2006). Optical Receiver. Retrieved from www.google.com/patents/US 7050724B1 $22^{\text {rd }}$ Jan; 2018.

[6] Janis, (2010). Free Space Communication System. Retrieved from www.google.co.in /patents/Ep2178227A1) $5^{\text {th }} \mathrm{Feb} ; 2018$.

[7] Sowmya, G., Dr. Subramanyan, M.V., \& Sofia, P. J. (2012). Design of an Optical Receiver using Tran-Impedance Amplifier with Light Detector and Equalization in MOS Technology. International Journal of Computer Trends and Technology-volume3issue3-2012. 
[8] Aashima, B., \& Gaurav, S. (2015). Performance Analysis of Optical Communication System Using Fiber Bragg Grafting. SSRG International Journal of Electronics and communication Engineering (SSRG-IJECE)-volume 2 issue1 Jan 2015.

[9] Deepak, B., Sumit, V., Sunaina, S., \& Swapnil, T. (2011). Free-Scale Semiconductor-Test and Measurement World. Retrieved from https://www.businesswire.com/news /home $/ 20050317005525 /$ en. $29^{\text {th }}$ Jan; 2018.

[10] Abdelhakim, B., Asmaa, O., \& Belabbes, S. (2015). Introduction to the Optical Communications by Simulating an Optical High Debit Transmission Chain Using OptiSystem with Comparison of Optical Windows. International Journal of Computer of Networks and communications security. Vol. 3, No. 2, pp. 8-15.

[11] Elechi, P., Orike, S., Minah-Eeba, W., \& Ikpo, C.E. (2018).
'Sensitivity Performance Analysis of Avalanche Photodiode and Pin Diode Detectors in Optical Receivers", Faculty of Engineering International Conference, Nnamdi Azikiwe University, Awka, 13-14 ${ }^{\text {th }}$ August, 2018.

[12] Elechi, P. \& Alalibo, T.A. (2017). Spectral Efficiency Analysis of GSM Networks in South-South Nigeria, European Journal of Engineering Research and Science, vol. 2, No. 6, pp. 7-11.

[13] Kang, J., Marhic, M.E., Li, B., Wang, X., \& Wong, K.K.Y. (2017) Optical Receiver sensitivity Enhancement by Singleand Dual-Band Fiber Optical Parametric Amplifier, Optic Express Journal, vol. 25, No. 22, pp. 27785-27794.

[14] Ali, H. (2017). Modeling and Simulation of High Speed Optical Fiber Communication System with OFDM, American Research Scientific Journal for Engineering, Technology and Sciences. Vol. 18, pp 1-8. 